\title{
THE SYRACUSE MEETING OF SECTION A AMERICAN ASSOCIATION FOR THE ADVANCEMENT OF SCIENCE
}

Section A (Mathematics) of the American Association for the Advancement of Science held sessions in the morning and afternoon of Tuesday, June 21st, 1932, at Syracuse, New York. About forty of those attending lunched together between the sessions at the University Cafeteria, drove to Green Lake in the late afternoon, and attended a picnic dinner.

At a joint session of Sections $A$ and $Q$ on Wednesday afternoon, Professor H. T. Davis of the University of Indiana presiding, Professor E. R. Hedrick of the University of California at Los Angeles showed how certain factors in the improvement of the teaching of mathematics depend on the proper cooperation between educationalists and mathematicians.

Among the other features of special interest to mathematicians were the report on the collegiate mathematics needed in the social sciences, presented at the Wednesday morning joint session of Section K and the Econometric Society, and the paper by Professor W. B. Carver of Cornell University on Mathematics and the advancement of science, given at the general session on Wednesday afternoon.

At the Tuesday morning session of Section A, Professor E. R. Hedrick, the retiring vice-president, presiding, papers were given by Professor H. M. Gehman of the University of Buffalo on Homeomorphic geometry of the projective plane, and by Professor W. A. Hurwitz of Cornell University on Logical foundations for groups and fields. At the afternoon session, Professor Virgil Snyder presiding, Professor J. A. Shohat of the University of Pennsylvania presented a paper on Interpolation.

Professor Gehman showed that analysis situs could be thought of as a branch of geometry in which the group of transformations of space is the group of homeomorphisms. The invariant properties of the following spaces were investigated: euclidean $n$-space, the euclidean plane, the inversion plane, the sphere, the projective plane. It was shown that the group of homeomorphisms of the projective plane which leave invariant the line at infinity form only a proper subgroup of the group of homeomorphisms of the euclidean plane, thus reversing the 
situation that exists under the group of projective transformations. Finally he stated a theorem which gives the distinguishing characteristics of a transformation of this subgroup.

Professor Hurwitz surveyed various forms of definition of groups, abelian groups, and fields, by sets of postulates. Sets of as few as three postulates for groups have been given; in some cases the undefined operation representing the direct combination $a b$ of elements of the group, in others the inverse combination $a b^{-1}$. For abelian groups, sets of as few as two postulates can be given, and for fields as few as four.

Professor Shohat dealt with various polynominal and trigonometric interpolation formulas, in the real and complex domains, emphasizing the two important properties of convergence (the function $f(x)$ to be interpolated assumed to be continuous) and stability, that is, the extent to which errors involved in the given values of $f(x)$ affect the interpolating function. (The degree of convergence was not discussed.)

For Lagrange's interpolating polynominal ( $\equiv L . I . P$.) in the real domain, say $-1 \leqq X \leqq 1$, he showed that (1) it is impossible to choose an infinite system of nodes in $(-1,1)$ so that the corresponding $L$. I. $P$. will be convergent for any continuous $f(x)$ over the whole interval, the best that can be obtained being convergence in case $f(x)$ satisfies a Dini-Lipschitz condition; and (2) the Lagrange interpolation formula is not stable. Dunham Jackson's trigonometric interpolation formula and Fejér's interpolating polynomial-a modification of $L$. I. P.-were both found to be stable and convergent for any continuous $f(x)$.

In the complex domain he discussed various $L$. I. $P$., the nodes being suitably chosen-coinciding with the zeros of either orthogonal Tchebycheff polynominals, or Faber-Tchebycheff polynomials of best approximation, or, in the case of circular regions, coinciding with vertices of regular inscribed polygons. In all these cases convergence holds for an analytic $f(x)$.

Returning to the real domain he discussed a polynomial formula due to $\mathrm{S}$. Bernstein which, although not an interpolation formula, employs a certain set of values of $f(x)$, has a very simple structure, and enjoys the two important propertiesconvergence for any continuous $f(x)$ and stability.

\section{F. F. Decker, Acting Secretary}

